\title{
Dynamic Correlations in a Random Spin-1/2 XY Chain
}

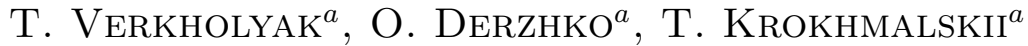 \\ AND J. STOLZE ${ }^{b}$ \\ ${ }^{a}$ Institute for Condensed Matter Physics, NASU \\ 1 Svientsitskii Street, L'viv-11, 79011, Ukraine \\ ${ }^{b}$ Institut für Physik, Universität Dortmund, 44221, Dortmund, Germany
}

We examine dynamic quantities of a random spin-1/2 isotropic $X Y$ chain in a transverse field. The randomness is related to the sign of the nearest-neighbor exchange interaction and can be eliminated by a suitable transformation. As a result, the dynamic quantities for the random spin chain are related to the same dynamic quantities for the homogeneous spin chain. We use the available results for the latter model to discuss the effect of randomness on the dynamic structure factors of the quantum spin chain.

PACS numbers: 75.10.Jm, 75.40.Gb

\section{Introduction}

Random quantum spin chains have been intensively investigated recently. The interplay between disorder and quantum fluctuations may produce intriguing properties of such systems. While a lot of theoretical efforts have gone into the random spin- $1 / 2 X X Z$ chain, a simpler model, the random spin- $1 / 2 X X 0$ (i.e. isotropic $X Y$ or $X X)$ chain, has received less attention although it may be of interest and importance since it admits in some cases a rigorous analysis. Our aim in this work is to examine the dynamic quantities of a random spin- $1 / 2 X X$ chain in a transverse field.

\section{Theory}

We consider $N$ spins $1 / 2$ in a row with the Hamiltonian

$$
H=\sum_{n=1}^{N}\left[\lambda_{n} J\left(s_{n}^{x} s_{n+1}^{x}+s_{n}^{y} s_{n+1}^{y}\right)+\Omega s_{n}^{z}\right]
$$

with periodic boundary conditions for convenience. (The boundary conditions become irrelevant as $N \rightarrow \infty$.) $\quad \lambda_{n} J$ is the $X X$ exchange interaction between 
the neighboring sites $n$ and $n+1$ and $\Omega$ is the external transverse $(z)$ magnetic field. We assume $\left\{\lambda_{n}\right\}$ to be a sequence of independent random variables each with the bimodal probability distribution $p\left(\lambda_{n}\right)=p \delta\left(\lambda_{n}+1\right)+(1-p) \delta\left(\lambda_{n}-1\right)$, $0 \leq p \leq 1$. This means that the $X X$ exchange interaction acquires randomly either the value $-J$ (with probability $p$ ) or the value $+J$ (with probability $1-p$ ). We are interested in random-averaged quantities and denote the averaging as $\overline{(\ldots)}=\prod_{n=1}^{N} \int_{-\infty}^{\infty} \mathrm{d} \lambda_{n} p\left(\lambda_{n}\right)(\ldots)$. This model was considered earlier [1, 2] although not for analysis of dynamic correlations. Below we focus on the dynamic structure factors

$$
\overline{S_{\alpha \beta}(\kappa, \omega)}=\frac{1}{N} \sum_{j=1}^{N} \sum_{m=1}^{N} \exp (-\mathrm{i} \kappa m) \int_{-\infty}^{\infty} \mathrm{d} t \exp (\mathrm{i} \omega t) \overline{\left\langle\Delta s_{j}^{\alpha}(t) \Delta s_{j+m}^{\beta}\right\rangle},
$$

where $\alpha, \beta=x, y, z,\langle(\ldots)\rangle=\operatorname{Tr}(\exp (-\beta H)(\ldots)) / \operatorname{Tr} \exp (-\beta H), \Delta s_{j}^{\alpha}(t)=$ $s_{j}^{\alpha}(t)-\left\langle s_{j}^{\alpha}\right\rangle, s_{j}^{\alpha}(t)=\exp (\mathrm{i} H t) s_{j}^{\alpha} \exp (-\mathrm{i} H t)$.

Remarkably, the introduced randomness can be eliminated from the Hamiltonian (1) by the gauge transformation

$$
s_{n}^{x} \rightarrow \tilde{s}_{n}^{x}=\lambda_{1} \ldots \lambda_{n-1} s_{n}^{x}, \quad s_{n}^{y} \rightarrow \tilde{s}_{n}^{y}=\lambda_{1} \ldots \lambda_{n-1} s_{n}^{y}, \quad s_{n}^{z} \rightarrow \tilde{s}_{n}^{z}=s_{n}^{z},
$$

after which the considered Hamiltonian $H$ (1) with the exchange constants $\lambda_{n} J$ transforms into the Hamiltonian $\tilde{H}$ of the homogeneous model with the exchange constant $J$ (up to an inessential boundary term). According to (3) the $z z$ dynamic structure factor (as well as all thermodynamic quantities) does not feel a random sequence $\left\{\lambda_{n}\right\}$. In contrast, the $x x$ and $x y$ dynamic structure factors do depend on $\left\{\lambda_{n}\right\}$.

Exploiting the gauge transformation (3) we find

$$
\begin{aligned}
& \overline{\left\langle s_{j}^{\alpha}(t) s_{j+m}^{\beta}\right\rangle}=(1-2 p)^{|m|}\left\langle\tilde{s}_{j}^{\alpha}(t) \tilde{s}_{j+m}^{\beta}\right\rangle \\
& = \begin{cases}\exp \left(-\frac{|m|}{\xi}\right)\left\langle\tilde{s}_{j}^{\alpha}(t) \tilde{s}_{j+m}^{\beta}\right\rangle, & 0 \leq p \leq \frac{1}{2}, \\
(-1)^{m} \exp \left(-\frac{|m|}{\xi}\right)\left\langle\tilde{s}_{j}^{\alpha}(t) \tilde{s}_{j+m}^{\beta}\right\rangle, & \frac{1}{2} \leq p \leq 1,\end{cases}
\end{aligned}
$$

where $\alpha, \beta=x, y$ and the correlation length $\xi=-1 / \ln |1-2 p|$. As a result, the dynamic structure factor (2) becomes

$$
\overline{S_{\alpha \beta}(\kappa, \omega)}=\sum_{m=0, \pm 1, \pm 2, \ldots} \exp \left(-\mathrm{i} \kappa m-\frac{|m|}{\xi}\right) \int_{-\infty}^{\infty} \mathrm{d} t \exp (\mathrm{i} \omega t)\left\langle\tilde{s}_{j}^{\alpha}(t) \tilde{s}_{j+m}^{\beta}\right\rangle(5)
$$

for $0 \leq p \leq 1 / 2$. (We notice that for the model (1) $\left\langle s_{n}^{x}\right\rangle=\left\langle s_{n}^{y}\right\rangle=0$.) If $1 / 2 \leq p \leq 1$, a factor $(-1)^{m}$ should be inserted under the sum in (5) (see (4)) and $\overline{S_{\alpha \beta}(\kappa, \omega)}$ for $1 / 2 \leq p \leq 1$ equals $\overline{S_{\alpha \beta}(\kappa \mp \pi, \omega)}$ for $0 \leq p \leq 1 / 2$. We use (5) to compute $\overline{S_{\alpha \beta}(\kappa, \omega)}$ through the known results for $\left\langle\tilde{s}_{j}^{\alpha}(t) \tilde{s}_{j+m}^{\beta}\right\rangle$ for the homogeneous chain obtained analytically or numerically (see [3] and references therein).

Let us consider the strong-field zero temperature case $|\Omega|>|J|$ and $\beta \rightarrow \infty$ when the dynamic structure factors $\tilde{S}_{\alpha \beta}(\kappa, \omega)$ are as follows: $\tilde{S}_{x x}(\kappa, \omega)=$ $\operatorname{isgn}(\Omega) \tilde{S}_{x y}(\kappa, \omega)=(\pi / 2) \delta(\omega-|\Omega|-J \cos \kappa)[4]$. As a result, after some calcula- 
tions we find

$$
\begin{aligned}
& \overline{S_{x x}(\kappa, \omega)}=\frac{\theta(\omega-|\Omega|+|J|) \theta(|\Omega|+|J|-\omega)}{2 \sqrt{J^{2}-(\omega-|\Omega|)^{2}}} \\
& \times \frac{J \sinh \frac{1}{\xi}\left[J \cosh \frac{1}{\xi}-(\omega-|\Omega|) \cos \kappa\right]}{\left(\omega-|\Omega|-J \cosh \frac{1}{\xi} \cos \kappa\right)^{2}+J^{2} \sinh ^{2} \frac{1}{\xi} \sin ^{2} \kappa}
\end{aligned}
$$

for $0 \leq p \leq 1 / 2$. $\overline{S_{x x}(\kappa, \omega)}$ for $1 / 2 \leq p \leq 1$ follows from (6) after the change $\kappa \rightarrow \kappa \mp \pi$. Equation (6) contains the above-mentioned exact result [4] in the nonrandom limit $\xi \rightarrow \infty$ (when $p=0$ or $p=1$ ). In the opposite limit of strong disorder $\xi=0$ (when $p=1 / 2$ ) the right hand side of (6) transforms into $\theta(\omega-|\Omega|+|J|) \theta(|\Omega|+|J|-\omega) /\left(2 \sqrt{J^{2}-(\omega-|\Omega|)^{2}}\right)$, i.e. $\overline{S_{x x}(\kappa, \omega)}$ contains the contribution of only the autocorrelation function (as it should be since $\xi$ tends to zero) and since in that limit $\left\langle\tilde{s}_{j}^{x}(t) \tilde{s}_{j}^{x}\right\rangle=\frac{1}{4 N} \sum_{\kappa} \exp \left(-\mathrm{i} \Lambda_{\kappa} t\right), \Lambda_{\kappa}=\Omega+J \cos \kappa$ [4] we have $\overline{S_{x x}(\kappa, \omega)}=\left(\frac{\pi}{2}\right) \rho(\omega)$ where $\rho(E)=\frac{1}{N} \sum_{\kappa} \delta\left(E-\Lambda_{\kappa}\right)$ is the density of states of elementary excitations (Jordan-Wigner spinless fermions). For other values of $p$ the dynamic structure factor $\overline{S_{x x}(\kappa, \omega)}(6)$ is restricted to the frequency region $|\Omega|-|J|<\omega<|\Omega|+|J|$ and shows square-root singularities as $\omega \rightarrow|\Omega| \pm|J|$. The frequency profiles at fixed $\kappa$ are similar (but not identical) to Lorentzian shapes centered at $\omega=|\Omega|+J \cosh (1 / \xi) \cos \kappa$ with line width $\Gamma=|J \sinh (1 / \xi) \sin \kappa|$.

\section{Numerical results and conclusions}

For arbitrary values of temperature and field we calculate $\overline{S_{x x}(\kappa, \omega)}$ using (5) and numerical results for the homogeneous chain [3]. Our findings are collected in Fig. 1. As $p$ slightly deviates from 0 (part (a)) the $x x$ dynamic structure factor shows behavior typical of the antiferromagnetic $X X$ chain. For $p$ around $1 / 2$ (parts (c), (d)) $\overline{S_{x x}(\kappa, \omega)}$ is completely determined by the autocorrelation function and similarly to the case examined above analytically the gray-scale plots show $\kappa$-independent stripes at $\omega=|J| \pm|\Omega|$. As $p$ approaches 1 the $x x$ dynamic structure factor shows behavior typical of the ferromagnetic $X X$ chain.

To summarize, we have considered a random spin- $1 / 2 X X$ chain, the dynamic properties of which can be examined rigorously using existing results for the dynamic quantities of the uniform spin- $1 / 2 X X$ chain. We note that the effect of temperature and of random couplings on the $x x$ dynamic structure factor are different. Although in both cases the autocorrelation function alone determines the $x x$ dynamic structure factor (for sufficiently high temperature or sufficiently strong randomness), at high temperature $S_{x x}(\kappa, \omega)$ is $\kappa$-independent and shows a single Gaussian ridge at $\omega=|\Omega|$. That is due to the Gaussian time decay of the autocorrelation function [5] which should be contrasted to the slow long-time decay of the autocorrelation function at low temperatures. Finally, we stress that 


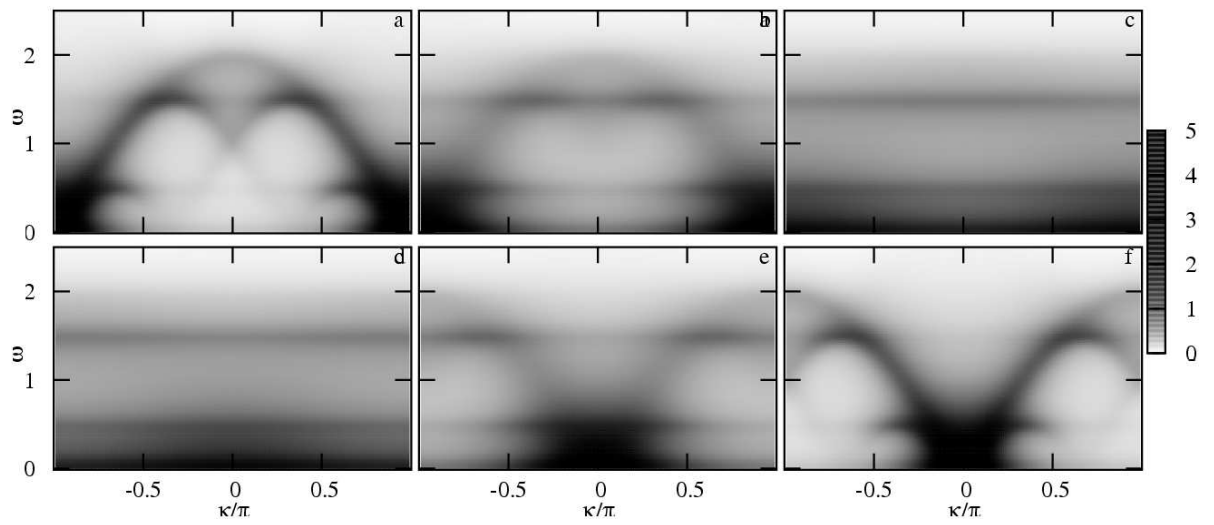

Fig. 1. $\overline{S_{x x}(\kappa, \omega)}$ for the spin-1/2 $X X$ chain with random-sign exchange interaction $(J=1, \Omega=0.5)$ at low temperature $(\beta=20)$. The values of $p$ are as follows: $p=0.1$ $(\xi \approx 4.48)(\mathrm{a}), p=0.25(\xi \approx 1.44)(\mathrm{b}), p=0.45(\xi \approx 0.43)(\mathrm{c}), p=0.55(\xi \approx 0.43)(\mathrm{d})$, $p=0.75(\xi \approx 1.44)(\mathrm{e}), p=0.9(\xi \approx 4.48)(\mathrm{f})$.

a direct brute-force approach implying repeated calculation of dynamic quantities for a certain realization of random couplings and then averaging the result over realizations of disorder requires too much computer time. Some possible extensions of the results reported here to other inhomogeneous quantum spin chains will be discussed elsewhere [6].

\section{Acknowledgments}

NATO support is acknowledged (CBP.NUKR.CLG 982540).

\section{References}

[1] A. Furusaki, M. Sigrist, P. A. Lee, K. Tanaka, N. Nagaosa, Phys. Rev. Lett. 73, 2622 (1994).

[2] K. Hamacher, J. Stolze, W. Wenzel, Phys. Rev. Lett. 89, 127202 (2002).

[3] O. Derzhko, T. Krokhmalskii, J. Stolze, J. Phys. A 33, 3063 (2000); O. Derzhko, T. Krokhmalskii, J. Stolze, J. Phys. A 35, 3573 (2002).

[4] H.B. Cruz, L.L. Gonçalves, J. Phys. C 14, 2785 (1981).

[5] U. Brandt, K. Jacoby, Z. Phys. B 25, 181 (1976).

[6] T. Verkholyak, O. Derzhko, T. Krokhmalskii, J. Stolze, in preparation. 